\title{
Análise bidimensional do processo de fusão de um material de mudança de fase em uma cavidade retangular
}

Two-dimensional analysis of the melting process of a phase change material in a rectangular cavity

\author{
R. S. Borahel; J. F. Raymundo Júnior; R. C. Oliveski \\ Escola Politécnica/Programa de Pós Graduação da Engenharia Mecânica, Universidade do Vale do Rio dos Sinos \\ (Unisinos), 93022-000, São Leopoldo - Rio Grande do Sul, Brasil
}

decesaroo@gmail.com

(Recebido em 11 de setembro de 2014; aceito em 29 de dezembro de 2014)

\begin{abstract}
Os materiais de mudança de fase podem ser utilizados em sistemas de armazenamento térmico de diferentes áreas da indústria, desta forma, a sua utilização nos sistemas de armazenamento de energia térmica apresenta-se como uma alternativa interessante para a otimização de uma grande gama de processos industriais. Levando em conta o aspecto inovador da utilização dos materiais de mudança de fase para armazenar/liberar energia na forma de calor latente, objetivou-se neste estudo, analisar numericamente o processo de fusão do eritritol contido em uma cavidade retangular, isto levando em conta a influência de variáveis geométricas, bem como, a temperatura a qual o sistema está submetido. O software comercial ANSYS FLUENT-14 foi utilizado para a execução de todas as simulações numéricas, em regime transiente, necessárias para a resolução do problema apresentado. Diferentes critérios de convergência foram adotados em todas as simulações realizadas. Dois tipos de validação numérica foram aqui realizadas, sendo estas: validação qualitativa e validação quantitativa. Resultados obtidos demonstram que a altura da cavidade não influencia de forma significativa o tempo de derretimento do material utilizado, contudo, uma grande influência da temperatura da parede de aquecimento foi encontrada no tempo necessário para a completa fusão do material.
\end{abstract}

Palavras-chave: materiais de mudança de fase, armazenamento de energia térmica, eritritol.

The phase change materials can be used in thermal storage systems in different fields of industry, thus its use in the thermal energy storage system is presented as an interesting alternative for optimizing a wide range of industrial processes. Considering the innovative aspect of the use of phase change materials for storing / releasing energy in the form of latent heat, the aim of this study was to analyze numerically the melting process of erythritol contained in a rectangular cavity, this considering the influence of geometric variables, as well as, the temperature at which the system is subjected. The ANSYS FLUENT-14, commercial software, was used to perform all numerical simulations in transient regime, these being necessary to the resolution of the studied problem. Different convergence criteria were adopted in all simulations. Two types of numerical validation has been performed here, these being: qualitative validation and quantitative validation. The results obtained in this work demonstrate that the height of the cavity does not influence significantly the melting time of the material used, however, a large influence of the wall temperature of heating was found in the time required for complete melting of the material. Keywords: phase change materials, thermal energy storage, erythritol.

\section{INTRODUÇÃO}

A utilização dos sistemas de armazenamento de energia térmica apresenta-se como uma alternativa interessante para a otimização de uma grande gama de processos, isto ocorrendo em diversas áreas industriais, tais como, as indústrias petroquímicas e de alimentos. Sistemas convencionais de armazenamento de energia térmica baseiam-se na utilização de materiais que funcionam de forma passiva, ou seja, materiais que acumulam energia sob a forma de calor sensível, isto ocorrendo através de suas capacidades caloríficas. Contudo, tais sistemas estão sendo lentamente substituídos por sistemas de armazenamento inovadores, estes utilizando os materiais de mudança de fase (PCMs) para absorver/liberar energia na forma de calor latente. Ou seja, o acumulo de energia ocorre na troca de fase do referido material, isto ocorrendo a uma 
temperatura constante. A quantidade de calor que pode ser absorvido/liberado por estes materiais é diretamente proporcional a sua entalpia de fusão, esta devendo ser a mais elevada possível para que se possa armazenar/liberar um máximo de energia com um mínimo de massa.

De acordo com Rathod e Banerjee [1], os materiais de mudança de fase atualmente disponíveis são classificados como: orgânicos (parafinas e não-parafinas), não orgânicos (metálicos e sais hidratados) e eutéticos (mistura de materiais orgânicos e/ou inorgânicos). Em relação aos materiais orgânicos, as parafinas destacam-se por serem os materiais mais utilizados para o armazenamento de energia térmica. Tal utilização ocorre devido a inúmeros fatores, tais como: elevada entalpia de fusão, variada temperatura de mudança de fase, inércia química e baixo custo financeiro se comparado ao das não-parafinas (Rathod e Banerjee [1]). Contudo, tais materiais não podem ser utilizados em sistemas submetidos a elevadas temperaturas (acima de $100{ }^{\circ} \mathrm{C}$ ), isto devido a estes materiais possuírem um ponto de fusão relativamente baixo, o mais elevado sendo próximo aos $96{ }^{\circ} \mathrm{C}$. Portanto, os sistemas de armazenamento de energia térmica submetidos a altas temperaturas não utilizam as parafinas como materiais de mudança de fase, mas sim as não-parafinas e os sais hidratados.

Como dito anteriormente, os materiais de mudança de fase podem ser utilizados em sistemas de armazenamento térmico de diferentes áreas da indústria, desta forma, são inseridos em sistemas que são submetidos a diferentes condições de operação. Contudo, tais condições de operação, bem como, o fenômeno de mudança de fase, devem sem amplamente compreendidos, de forma que se possa desenvolver e aperfeiçoar estes sistemas inovadores. Portanto, objetivouse neste estudo, analisar numericamente o processo de fusão do eritritol contido em uma cavidade retangular bidimensional, isto com vistas ao desenvolvimento e otimização de tais sistemas.

\section{MATERIAL E MÉTODOS}

O modelo físico, modelo matemático, as condições de contorno utilizadas, bem como, outras informações que são relevantes para a reprodução deste estudo, são descritas nos subitens abaixo. Já a validação numérica do modelo aqui implementado, está apresentada no término desta seção.

\subsection{Modelo físico}

O sistema de armazenamento de energia térmica estudado neste trabalho é apresentado na Figura 1 (a-b). O referido sistema consiste de um canal vertical dividido em pequenas cavidades retangulares, tal divisão devido à inserção de aletas internas, sendo estas de alumínio, assim como, as paredes laterais do sistema. Deve-se atentar ao fato de que cada cavidade é preenchida pelo material de mudança de fase, este que troca calor com o fluido de transferência de calor (HTF) que se encontra escoando no lado externo do canal, sendo que tal troca ocorre através das paredes laterais do sistema. Em relação à temperatura destas paredes, pode-se considerá-la como sendo constante e igual à temperatura do HTF (água). Tal consideração se deve à pequena espessura das paredes, bem como, em razão da temperatura da água ser mantida constante, isto devido a uma elevada vazão mássica.

O sistema de armazenamento de energia térmica, apresentado na Figura 1 (a-b), foi considerado um problema bidimensional, desta forma, desconsiderou-se a espessuras das aletas (e), bem como, o espaçamento existente entre cada aleta $(W)$. Portanto, o domínio de cálculo deste estudo se trata de uma cavidade retangular bidimensional, esta sendo apresentada na Figura 1 (c). O problema aqui estudado levou em conta a influência da altura da cavidade no processo de derretimento, desta forma, o domínio de cálculo apresentado foi estudado para três alturas distintas $(H)$, estas sendo: 10,20 e $30 \mathrm{~cm}$. Em relação à largura da cavidade $(B)$, esta foi mantida constante e igual a $0,5 \mathrm{~cm}$. Deve-se ainda salientar que apenas $85 \%$ da altura $(H)$ da cavidade foi preenchida pelo PCM, isto para permitir a livre expansão do mesmo durante a troca de fase. 


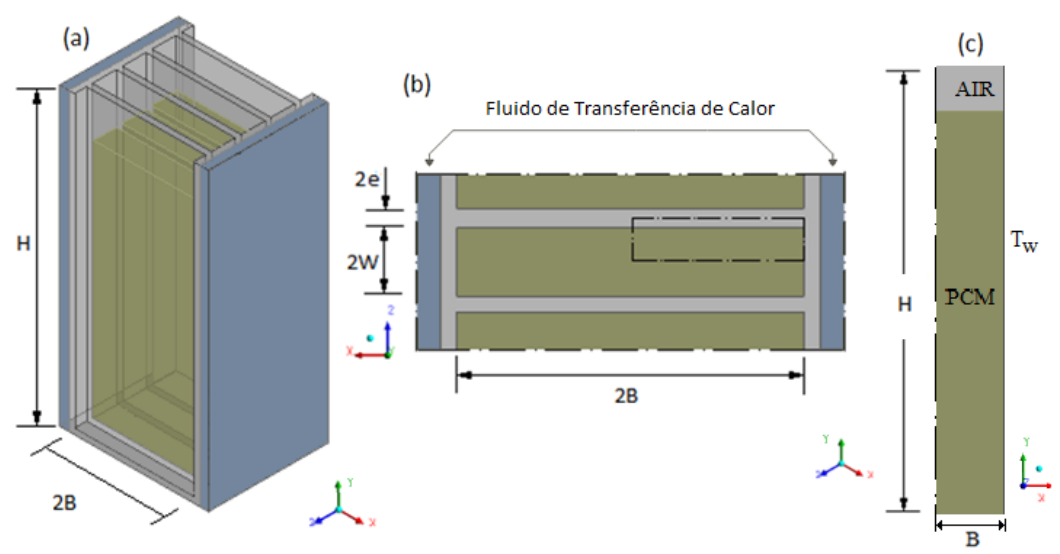

Figura 1: Geometria do Sistema de armazenamento de energia térmica: (a) geometria tridimensional; (b) visão superior da geometria tridimensional e (c) domínio computacional bidimensional.

Neste trabalho, optou-se pela utilização do eritritol como sendo o material de mudança de fase. Tal escolha ocorreu em virtude das temperaturas as quais o sistema de armazenamento de energia seria submetido, tais temperaturas sendo maiores que $100{ }^{\circ} \mathrm{C}$. Apesar da vasta pesquisa realizada, apenas três referências que mencionavam as propriedades físicas do eritritol foram encontradas, tais referências sendo os trabalhos de Agyenim et al. [2], Hesaraki [3] e Sillik e Gregson [4]. Os estudos de Agyenim et al. [2] e Hesaraki [3], mencionam uma variação linear de todas as propriedades do eritritol em função de sua temperatura. Contudo, valores referentes à viscosidade dinâmica, são apresentados apenas por Hesaraki [3], tal propriedade não sendo mencionada no estudo conduzido por Agyenim et al. [2]. Já Sillik e Gregson [4], apresentam resultados experimentais para a viscosidade do eritritol, tais resultados mostram que a variação da viscosidade em função da temperatura ocorre de forma logarítmica e não de forma linear como afirmado por Hesaraki [3]. Portanto, neste trabalho, optou-se pela utilização dos valores referentes à viscosidade dinâmica expressos no trabalho de Sillik e Gregson [4], assim como, os valores apresentados por Agyenim et al. [2] e Hesaraki [3] para as propriedades físicas restantes. Deve-se salientar que todas as propriedades físicas adotadas para o eritritol estão apresentadas na Tabela 1, bem como, as unidades referentes a cada propriedade.

Tabela 1: Propriedades físicas do eritritol.

\begin{tabular}{cc}
\hline Propriedades & \\
\hline Calor específico $\left(\mathbf{J ~ k g}^{-1} \mathbf{K}^{-1}\right)$ & $2250(389 \mathrm{~K}) ; 2740(413 \mathrm{~K})$ \\
Condutividade térmica $\left(\mathbf{W ~ m ~ m}^{-1} \mathbf{K}^{-\mathbf{1}}\right)$ & $0,733(389 \mathrm{~K}) ; 0,326(413 \mathrm{~K})$ \\
Entalpia de fusão $\left(\mathbf{J ~ k g}^{-1}\right)$ & 339800 \\
Massa específica $\left(\mathbf{~ k g ~ m}^{-3}\right)$ & $1480(389 \mathrm{~K}) ; 1300(413 \mathrm{~K})$ \\
Ponto de fusão $(\mathbf{K})$ & 391 \\
Viscosidade dinâmica $\left(\mathbf{k g ~ m}^{-1} \mathbf{~ s}^{-\mathbf{1}}\right)$ & $0,000027749 \mathrm{~T}^{2}-0,0231747 \mathrm{~T}+4,844$ \\
\hline
\end{tabular}

\subsection{Modelo matemático}

O escoamento da fase líquida de PCM foi assumido como sendo laminar, tal condição sendo adotada devido à baixa velocidade do escoamento, bem como, as pequenas dimensões da cavidade onde o mesmo se encontra armazenado. As equações governantes do sistema PCM-Ar, baseadas no método da abordagem entalpia-porosidade, são descritas por Ye et al. [5] e estão apresentadas logo abaixo:

$$
\frac{\mathrm{D} \alpha_{\mathrm{n}}}{\mathrm{Dt}}=0
$$




$$
\begin{aligned}
& \rho \frac{\mathrm{DV}}{\mathrm{Dt}}=-\nabla \mathrm{p}+\mu \nabla \overrightarrow{\mathrm{V}}+\rho \overrightarrow{\mathrm{g}}+\overrightarrow{\mathrm{S}} \\
& \frac{\mathrm{Dh}}{\mathrm{Dt}}=\mathrm{k} \nabla^{2} \mathrm{~T}
\end{aligned}
$$

onde: $\rho$ é a massa específica do PCM, $\alpha$ é a fração de volume média, $V$ é o vetor de velocidade, $p$ é a pressão, $\mu$ é a viscosidade dinâmica, $g$ é a gravidade, $S$ é o termo fonte, $h$ é a entalpia, $k$ é a condutividade térmica, $T$ é a temperatura e $t$ corresponde ao tempo.

Já a entalpia foi definida como a soma da entalpia sensível $\left(h_{s}\right)$ com a variação de entalpia devido à mudança de fase $(\gamma L)$, onde $h_{\text {ref }}$ corresponde à entalpia de referencia a uma temperatura de referência $\left(T_{r e f}\right)$ e $\gamma$ corresponde à fração de líquido durante a mudança de fase. Sendo que a mudança de fase ocorre em um intervalo de temperatura $\left(T_{s}<T<T_{l}\right)$, onde $T_{s}$ é a temperatura de solidificação e $T_{l}$ a temperatura de fusão, sendo tais afirmações definidas pelas seguintes relações (Shmueli et al. [6]):

$$
\begin{aligned}
& h_{s}=h_{\text {ref }}+\int_{\text {Tref }}^{\mathrm{T}} \mathrm{c}_{\mathrm{p}} \mathrm{dT} \\
& \gamma=\left\{\begin{array}{ccc}
0 & \text { if } & \mathrm{T}<\mathrm{T}_{\mathrm{s}} \\
1 & \text { if } & \mathrm{T}>\mathrm{T}_{1} \\
\frac{\mathrm{T}-\mathrm{T}_{\mathrm{s}}}{\mathrm{T}_{1}-\mathrm{T}_{\mathrm{s}}} & \text { if } & \mathrm{T}_{\mathrm{s}}<\mathrm{T}<\mathrm{T}_{1}
\end{array}\right.
\end{aligned}
$$

A região pastosa (região parcialmente solidificada) é tratada como um meio poroso pelo método da abordagem entalpia-porosidade. Deve-se salientar que a porosidade em cada elemento é definida como sendo igual à fração de líquido ali presente. Portanto, em regiões onde somente há PCM na fase sólida a porosidade será igual à zero, desta forma, extinguindo as velocidades nestas regiões. Outro fator que deve ser frisado nestas regiões é o termo fonte, este apresentado anteriormente na equação do momento (Eq. 2) e que acaba por tomar a seguinte forma devido à baixa porosidade encontrada nas referidas regiões (ANSYS-FLUENT [7]):

$$
S=\frac{C(1-\beta)^{2}}{\left(\beta^{3}+\varepsilon\right)}\left(\vec{V}-\vec{V}_{p}\right)
$$

onde: $\beta$ é a fração de volume do líquido, $\varepsilon$ é uma pequena constante (igual a 0,001 ) para não permitir uma divisão por zero, $C$ é a constante da zona pastosa e $V_{p}$ é definido como a velocidade da região pastosa. Pesquisado por diversos autores (Voller e Prakash [8], Brent et al. [9] e Shmueli et al. [6]), $C$ é definido como sendo uma constante que depende da morfologia da zona pastosa (Voller e Prakash [8]). Sendo que no presente trabalho numérico, um valor de $1 \mathrm{X} 10^{8}$ foi admitido para a tal constante.

As equações referentes à fração de líquido $(f l)$, assim como, ao fluxo de calor ( $q$ ”), foram desenvolvidas para uma cavidade retangular bidimensional e são apresentadas logo abaixo. Tais equações partiram das equações apresentadas por Shmueli et al. [6] para um tubo cilíndrico vertical.

$$
\mathrm{fl}=1-\frac{\mathrm{A}_{\mathrm{PCM}}(\mathrm{t})}{\mathrm{A}_{\mathrm{PCM}}(\mathrm{t}=0)}
$$




$$
q^{\prime \prime}=0,85 \rho L B \frac{f l}{t}
$$

onde: $A_{P C M}$ é a área ocupada pelo PCM na fase sólida.

\subsection{Condições iniciais e de contorno}

Condições de contorno do problema estudado são necessárias para a execução de uma simulação numérica por CFD (Computacional Fluid Dynamics). Tais condições, devendo ser inseridas no software utilizado para a definição das condições físicas do problema. Neste trabalho, uma grande gama de condições iniciais e de contorno foi utilizada, uma delas sendo a temperatura inicial do PCM (Ti), esta sendo igual a $389 \mathrm{~K}$. Vale salientar que a temperatura inicial do ar contido dentro da cavidade é igual à temperatura inicial do PCM, portanto, o ar também se encontra a $389 \mathrm{~K}$. Em relação à parede de aquecimento, três temperaturas $(T w)$ foram utilizadas, sendo elas: 401, 406 e $411 \mathrm{~K}$. Sendo assim, as simulações numéricas foram conduzidas para três diferenças de temperatura $(\Delta T)$, tais diferenças ocorrendo entre a temperatura de fusão do PCM e a temperatura da parede de aquecimento $(T w)$. Outra condição de contorno foi prescrita para a parede de aquecimento, bem como, para todas as paredes sólidas do domínio computacional [Figura 1 (c)], tal condição adotada foi a de não deslizamento. A base da cavidade retangular também foi considerada uma parede sólida, onde aplicou-se uma condição de fluxo de energia nulo, ou seja, a base da cavidade foi admitida como adiabática. Já no topo da cavidade, foi prescrita a condição de Pressure-Outlet, com pressão manométrica igual a $0 \mathrm{~Pa}$ e temperatura de escoamento reverso igual à $T w$. Em relação à parede remanescente, parede esquerda, condição de simetria foi adotada.

\subsection{Solução numérica e independência de malha}

Para alcançar os objetivos propostos neste trabalho, se fez necessário à utilização de diferentes softwares comerciais da ANSYS, sendo esta uma empresa especializada na criação e desenvolvimento de softwares voltados a resolução de problemas de engenharia. Desta forma, optou-se pela utilização do software comercial ANSYS FLUENT-14 para a execução de todas as simulações numéricas, em regime transiente, necessárias para a resolução do problema apresentado. Diferentes critérios de convergência foram adotados em todas as simulações realizadas, sendo aplicado um critério de $1 \times 10^{-8}$ para a equação da energia, bem como, um critério de $1 \mathrm{X}^{-6} 0^{-6}$ para a continuidade e as componentes da velocidade. Em relação ao intervalo de tempo utilizado, foi adotado um valor de $0,01 \mathrm{~s}$ com um número máximo de 1000 iterações por intervalo de tempo. As equações (1-6) foram resolvidas para cada elemento do domínio computacional, sendo que foi utilizado o esquema PRESTO para a equação da correção da pressão, PISO para o acoplamento da pressão-velocidade e Second Order Upwind para a solução das equações do momento e da energia.

A fim de garantir-se a independência de malha, ou seja, que os resultados aqui apresentados não sejam influenciados pela malha utilizada, três malhas numéricas com diferente número de elementos foram criadas no ICEM CFD (software de criação de geometrias e malhas da ANSYS) e testadas no domínio computacional. Sendo que a quantidade de elementos em cada uma das três malhas é aproximadamente: $7,3 \times 10^{3} ; 14 \times 10^{3}$ e $27 \times 10^{3}$ elementos. Deve-se ainda salientar que tais malhas bidimensionais são estruturadas, hexaédricas e refinadas próximo à parede de aquecimento, assim como, na interface PCM-Ar. Em relação aos testes realizados, resultados preliminares não mostraram diferenças significativas entre os resultados obtidos com as malhas de $14 \times 10^{3}$ e $27 \times 10^{3}$ elementos. Portanto, os resultados apresentados neste trabalho, foram obtidos por intermédio de uma malha com um número médio de elementos $\left(14 \mathrm{X} 10^{3}\right.$ elementos). Tal escolha ocorreu em virtude desta malha apresentar uma redução considerável no tempo necessário de simulação, isto se comparado ao tempo requerido para a obtenção dos resultados ao utilizar-se a malha com o maior número de elementos ( $27 \times 10^{3}$ elementos). 


\subsection{Validação numérica}

Uma validação numérica do modelo aqui apresentado foi realizada, tal procedimento foi efetuado com objetivo de garantir que o modelo apresentado é válido para o estudo numérico do processo de fusão de um material de mudança de fase. Portanto, o modelo anteriormente apresentado foi aplicado a um tubo cilíndrico vertical, isto de modo que se pudesse reproduzir o estudo realizado por Shmueli et al. [6]. O referido problema, apresentado por Shmueli et al. [6], e reproduzido para fins de validação numérica, consiste da fusão de um PCM, este sendo o RT27.

Dois tipos de validação são apresentados neste trabalho, estes sendo: validação qualitativa e quantitativa. A validação qualitativa se trata de uma comparação visual dos campos de massa específica, enquanto, a validação quantitativa se trata de uma comparação da fração de PCM fundido ao longo do tempo. Resultados referentes à validação qualitativa são apresentados na Figura 2 (a-c), isto para os instantes de 4, 20 e 36 minutos de aquecimento, respectivamente. Em relação aos resultados demonstrados na respectiva figura, estão os resultados numéricos (i) e experimentais (ii) de Shmueli et al. [6], assim como, os resultados numéricos obtidos neste trabalho (iii). Nesta figura, percebe-se claramente o espaço ocupado pelo ar (topo do domínio), bem como, as duas fases do RT27 (liquido e sólido). Também se percebe uma grande similaridade entre os dois resultados numéricos, porém, ambos apresentam uma pequena diferença se comparados ao experimental, especialmente no último instante apresentado.

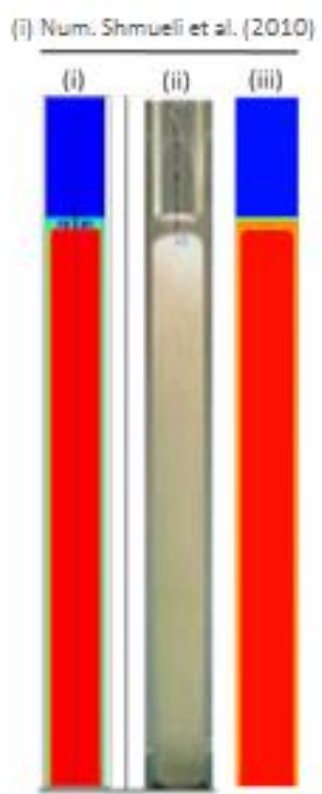

(a) $4 \mathrm{~min}$

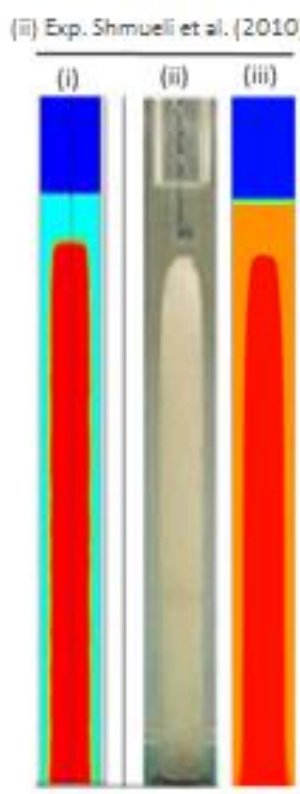

(b) $20 \mathrm{~min}$

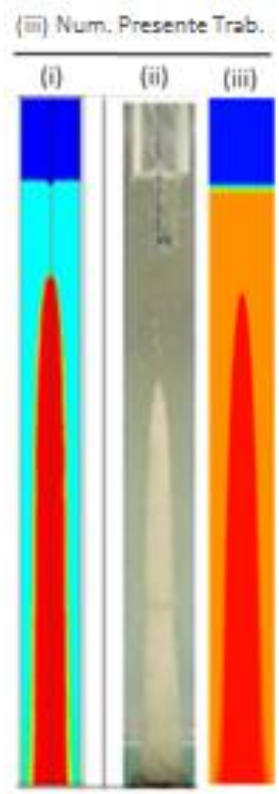

(c) $36 \mathrm{~min}$

Figura 2: Campo de densidade (a) 4 min; (b) 20 min e (c)36min. Resultados de: (i) numérico de Shmueli et al. (2010); (ii) experimental de Shmueli et al. (2010) e (iii) numérico do presente trabalho.

Já os resultados referentes à validação quantitativa estão apresentados na Figura 3, esta demonstrando a fração de PCM fundido (fração de líquido) ao longo do tempo. Desta forma, os resultados obtidos nos estudos conduzidos por Shmueli et al. [6], bem como, os resultados numéricos obtidos neste estudo, são apresentados nesta figura. Uma pequena diferença entre os resultados numéricos pode ser vista nos estágios iniciais e finais, porém, nenhuma diferença pode ser percebida no restante do tempo. Desta forma, pode-se dizer que o modelo matemático e numérico aqui apresentado é apropriado para o estudo do derretimento de um material de mudança de fase. 


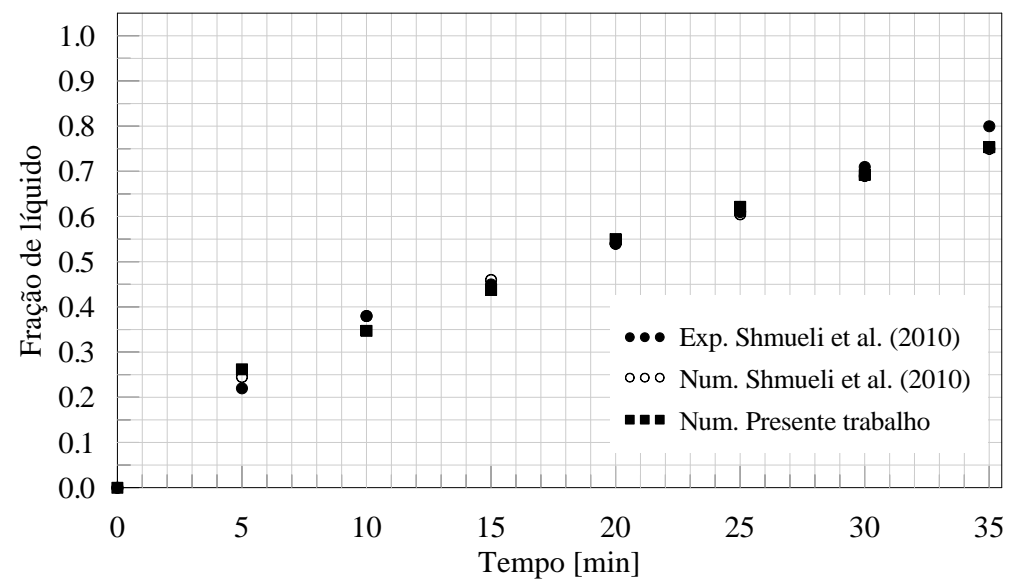

Figura 3: Comparação da fração de líquido ao longo do tempo.

\section{RESULTADOS E DISCUSSÃO}

Como relatado anteriormente, todos os resultados aqui apresentados foram obtidos por intermédio de simulações numéricas. Resultados referentes à fração de PCM fundido, bem como, o fluxo de calor médio ao longo do tempo, são apresentados nesta seção. A Figura 4 (a-c) ilustra a influência da altura da cavidade no tempo necessário para a completa fusão do PCM. Resultados referentes à fração de líquido (fração de PCM fundido), isto para as três alturas estudadas com um $\Delta T$ de $10{ }^{\circ} \mathrm{C}$, são apresentados na Figura 4 (a). Analisando os resultados mostrados na referida figura, nota-se que a altura da cavidade não influenciou no tempo necessário para obter-se uma fração de líquido igual a 0,99. Em contraste, diferenças de tempo para obter-se a completa fusão do material (fração de líquido igual a 1,0) foram encontradas para as três alturas analisadas, isto partindo de uma fração de líquido igual a 0,99 . Um comportamento semelhante da influência da altura foi verificado para os dois $\Delta T$ s restantes (15 e $20^{\circ} \mathrm{C}$ ), isto sendo apresentado na Figura 4 (b-c).
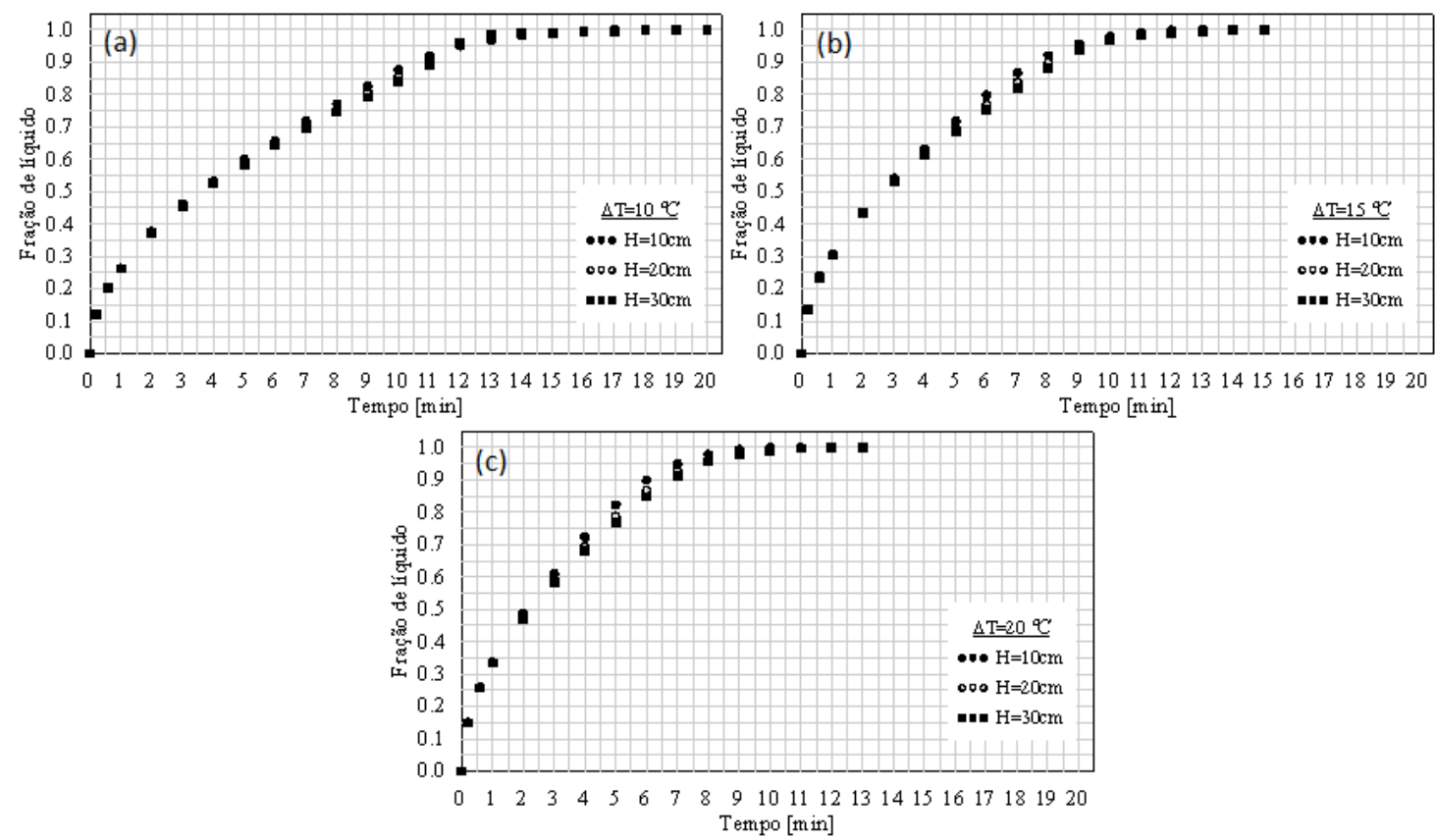

Figura 4: Fração de líquido ao longo do tempo para as três alturas analisadas com diferentes $\Delta T$ s:

(a) $\Delta T=10^{\circ} \mathrm{C}$; (b) $\Delta T=15^{\circ} \mathrm{Ce}$ (c) $\Delta T=20^{\circ} \mathrm{C}$. 
Em relação ao fluxo de calor médio, a altura da cavidade também não se mostrou influente neste fator. Os resultados obtidos [Figura 5 (a-c)] demonstram que o fluxo de calor médio é o mesmo para as três alturas estudadas, isto ocorrendo para um mesmo $\Delta T$.
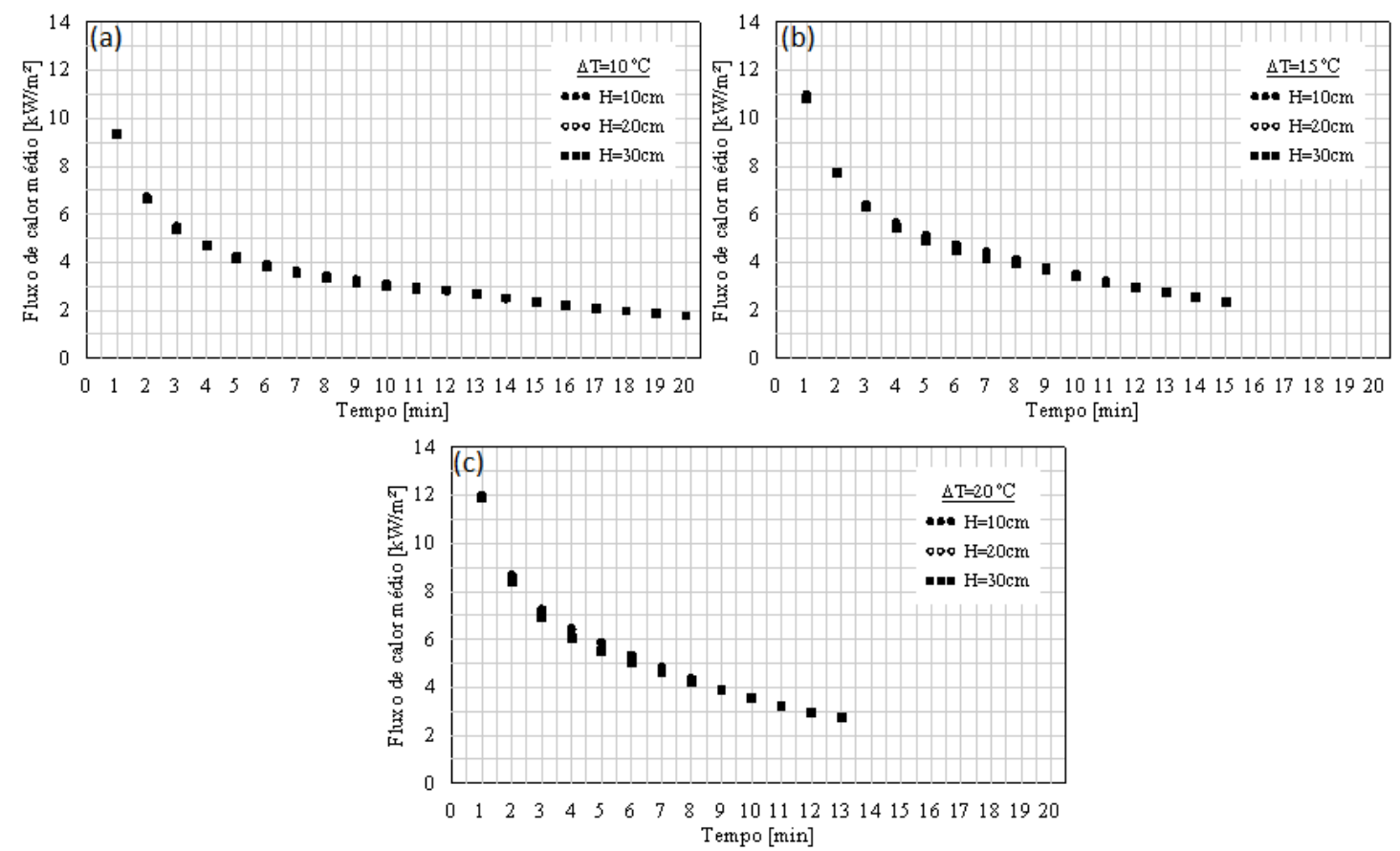

Figura 5: Fluxo de calor médio ao longo do tempo para as três alturas analisadas com diferentes $\Delta T$ :

(a) $\Delta T=10{ }^{\circ} \mathrm{C}$; (b) $\Delta T=15^{\circ} \mathrm{C} e$ (c) $\Delta T=20^{\circ} \mathrm{C}$.

Os resultados referentes ao comportamento da fração de líquido ao longo do tempo, isto para os três $\Delta T s$ utilizados, são apresentados na Figura 6 (a-c). Tais resultados demonstram que ao elevar-se a temperatura da parede de aquecimento $(T w)$, de $401 \mathrm{~K}$ para $411 \mathrm{~K}$, resulta em uma redução no tempo necessário para a completa fusão do PCM utilizado, sendo esta redução igual a 7 minutos. Deve-se ainda salientar que tal redução foi observada para todas as alturas estudadas.
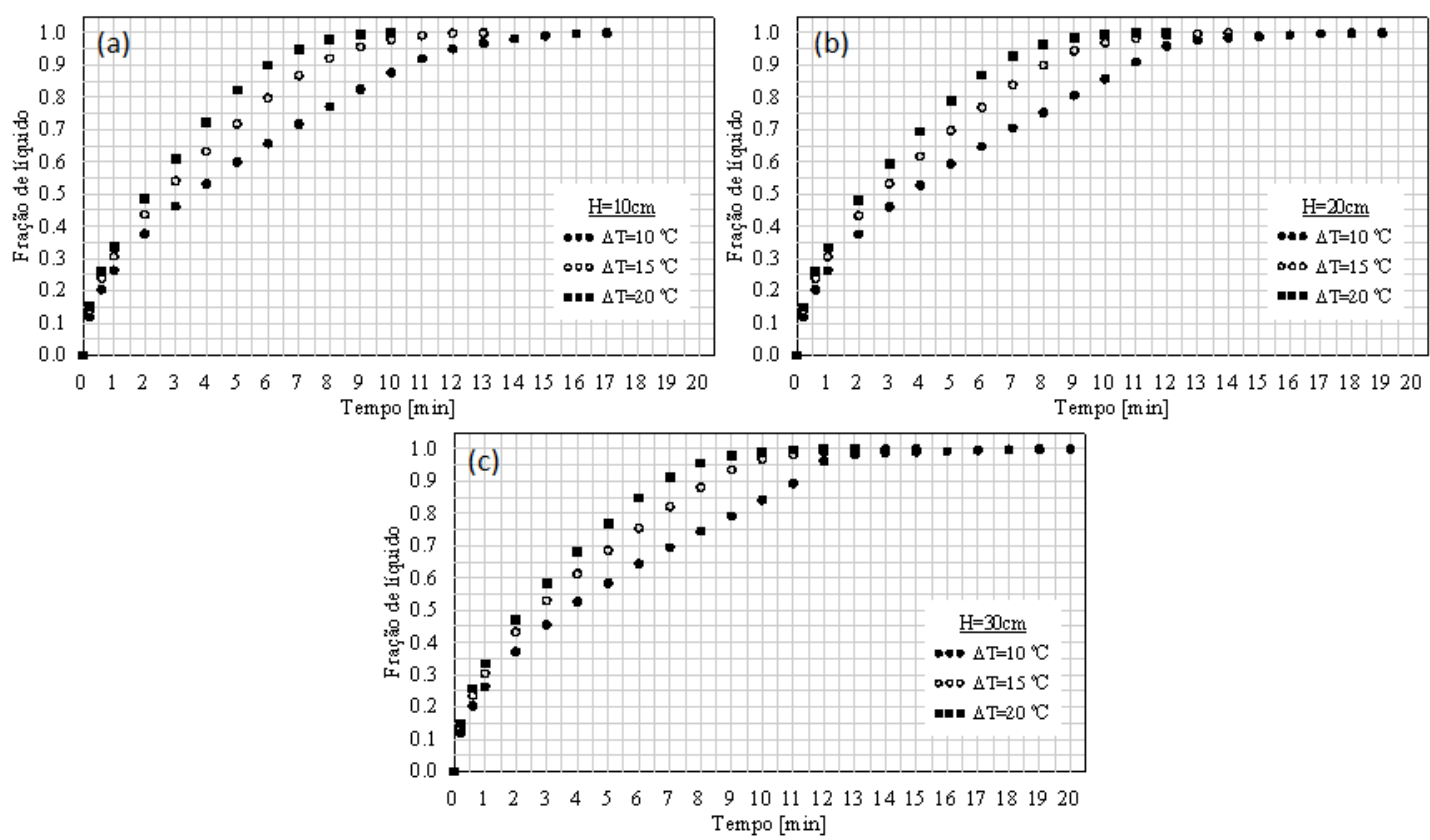

Figura 6: Fração de líquido ao longo do tempo para os três $\Delta T$ s estudados com diferentes alturas: (a) $H=10 \mathrm{~cm} ;$ (b) $H=20 \mathrm{~cm} \mathrm{e} \mathrm{(c)} H=30 \mathrm{~cm}$. 
A influência da temperatura da parede de aquecimento $(T w)$ no fluxo de calor médio ao longo do tempo também foi explorada neste trabalho, sendo que os resultados obtidos são ilustrados na Figura 7 (a-c). Uma diferença significativa entre os valores do fluxo de calor médio para os três $\Delta T s$ estudados, isto nos minutos iniciais de aquecimento, foi visualizada numa cavidade retangular com altura igual a $10 \mathrm{~cm}$ [Figura 7 (a)]. Tal comportamento também foi observado para as duas alturas remanescentes, sendo estas iguais a 20 e $30 \mathrm{~cm}$ [Figura 7 (bc)]. Também se observou que com o passar do tempo tal diferença diminui consideravelmente em todos os casos estudados, sendo que após 10 minutos já não se é mais possível observá-la.
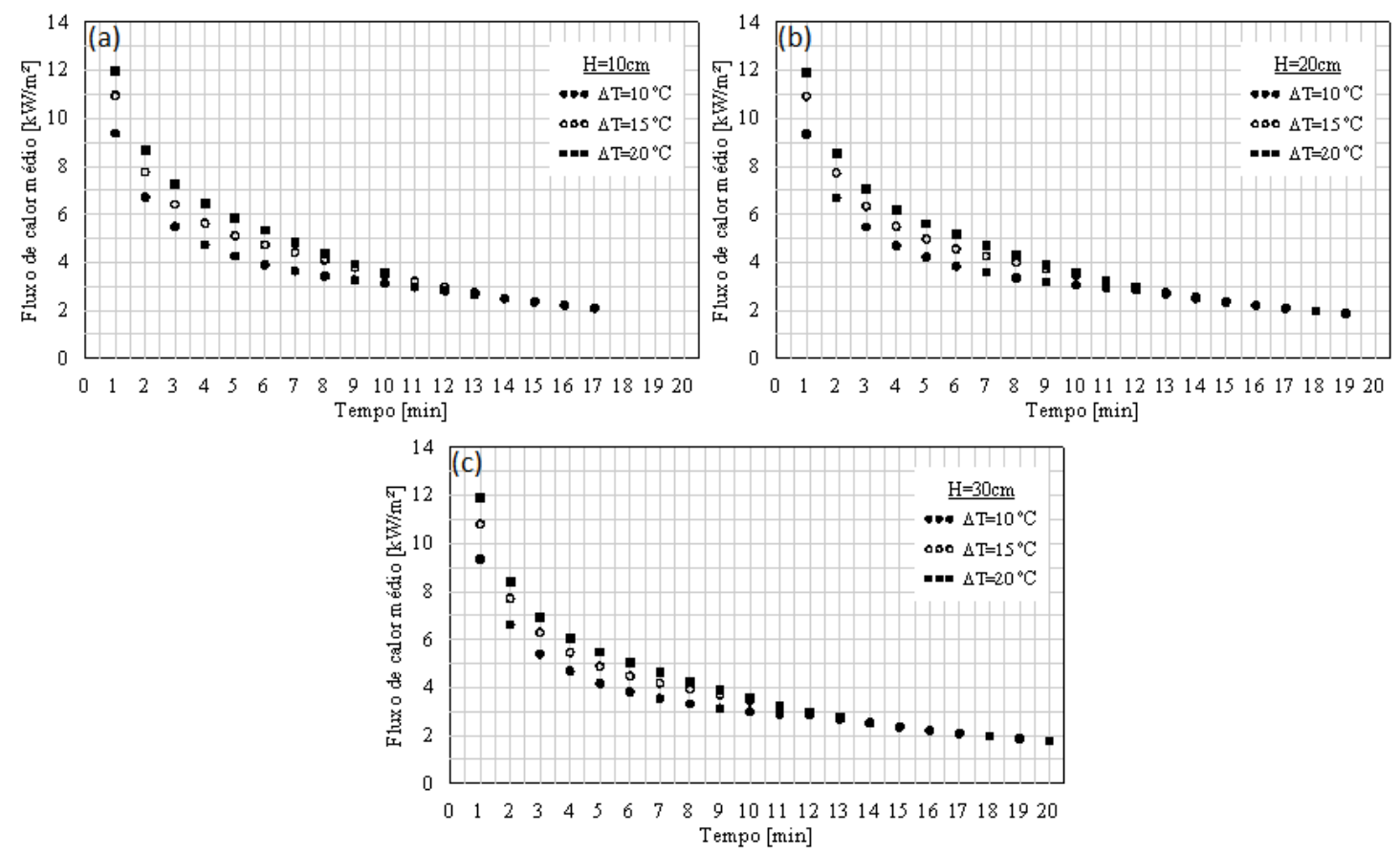

Figura 7: Fluxo de calor médio ao longo do tempo para os três $\Delta T$ s estudados com diferentes alturas: (a) $H=10 \mathrm{~cm}$; (b) $H=20 \mathrm{~cm}$ e (c) $H=30 \mathrm{~cm}$.

\section{CONCLUSÃO}

Como dito anteriormente, buscou-se neste trabalho avaliar a influência de fatores geométricos, bem como, a temperatura a qual o sistema está submetido, no processo de fusão do eritritol contido em uma cavidade retangular. Desta forma, realizaram-se diversas simulações numéricas com o software ANSYS FLUENT-14, nas quais analisou-se a influência da altura da cavidade, assim como da temperatura da parede de aquecimento. O domínio de cálculo foi estudado para três alturas distintas $(H)$, estas sendo: 10, 20 e $30 \mathrm{~cm}$. Em relação à parede de aquecimento, três temperaturas $(T w)$ foram utilizadas, sendo elas: 401, 406 e $411 \mathrm{~K}$. Resultados referentes à fração de líquido, isto para as três alturas estudadas, bem como, para os três $\Delta T s$ analisados, foram apresentados neste trabalho. Tais resultados demonstram que a altura da cavidade não influencia o tempo necessário para a completa fusão do PCM utilizado, contudo, uma grande dependência da fração de líquido em relação à temperatura da parede de aquecimento $(T w)$ foi observada. Em relação ao fluxo de calor médio, a altura da cavidade também não se mostrou influente neste fator, já que os resultados obtidos demonstram que o fluxo de calor médio é o mesmo para as três alturas estudadas, isto ocorrendo independente do $\Delta T$ utilizado. Já a temperatura da parede de aquecimento $(T w)$ se mostrou influente no fluxo de calor médio, principalmente nos instantes iniciais. Portanto, pode-se concluir que a temperatura a qual o sistema está submetido, assim como os parâmetros geométricos do sistema de armazenamento utilizado, devem ser considerados no projeto de construção de um sistema de armazenamento de energia térmica baseado na utilização de um material de mudança de fase. 


\section{AGRADECIMENTOS}

Os autores agradecem ao CNPq (Conselho Nacional de Desenvolvimento Científico e Tecnológico), à CAPES (Coordenação de Aperfeiçoamento de Pessoal de Nível Superior), à FAPERGS (Fundação de Ampara a Pesquisa do Rio Grande do Sul) e à Unisinos (Universidade do Vale do Rio dos Sinos), pelo suporte financeiro em diferentes etapas desta pesquisa.

\section{REFERÊNCIAS BIBLIOGRÁFICAS}

1. Rathod MK, Benerjee J. Thermal stability of phase change materials used in latent heat energy storage systems: A review. Renewable and Sustainable Energy Reviews. 2013 Feb;18:246-258, doi:10.1016/j.rser.2012.10.022.

2. Agyenim F, Eames P, Smyth M. Heat transfer enhancement in medium temperature thermal energy storage using a multitube heat transfer array. Renewable Energy. 2010 Jan;35(1):198-207, doi: 10.1016/renene.2009.03.010.

3. Hesaraki, A. CFD modelling of a heat charging process in a direct-contact container for mobilized thermal energy storage. Disponível em: http://www.divaportal.org/smash/get/diva2:430712/FULLTEXT02.

4. Sillick M, Gregson CM. Spray chill encapsulation of flavors within anhydrous erythritol crystals. LWT - Food Science and Technology. 2012 Sep;48(1):107-113, doi:10.1016/j.lwt.2012.02.022.

5. Ye WB, Zhu DS, Wang N. Fluid flow and heat transfer in a latent thermal energy unit with different phase change material (PCM) cavity volume fractions. Applied Thermal Engineering. 2012 Sep;42:49-57, doi:10.1016/j.applthermaleng.2012.03.002.

6. Shmueli H, Ziskind G, Letan R. Melting in a vertical cylindrical tube: Numerical investigation and comparison with experiments. International Journal of Heat and Mass Transfer. 2010 Sep;53(1020):4082-4091, doi:10.1016/j.ijheatmasstransfer.2010.05.028.

7. ANSYS FLUENT. Release 14.0: Solver modelling guide. 2011.

8. Voller VR, Prakash C. A fixed grid numerical modeling methodology for convection-diffusion mushy region phase-change problems. International Journal of Heat and Mass Transfer. 1987 Aug;30(8):1709-1719, doi:10.1016/0017-9310(87)90317-6.

9. Brent AD, Voller VR, Reid KJ. Enthalpy-porosity technique for modeling convection-diffusion phase change: Application to the melting of a pure metal. Numerical heat transfer. 1988;13(3):297-318, doi: 10.1080/10407788808913615. 